\title{
ÉTUDE DE REMĖDES AUX PULSATIONS DE PRESSION DANS LES CIRCUITS INDUSTRIELS DE GAZ COMPRIMÉ
}

par P. LEON

Ingénieur au Département des Applications Industrielles SOGREAH

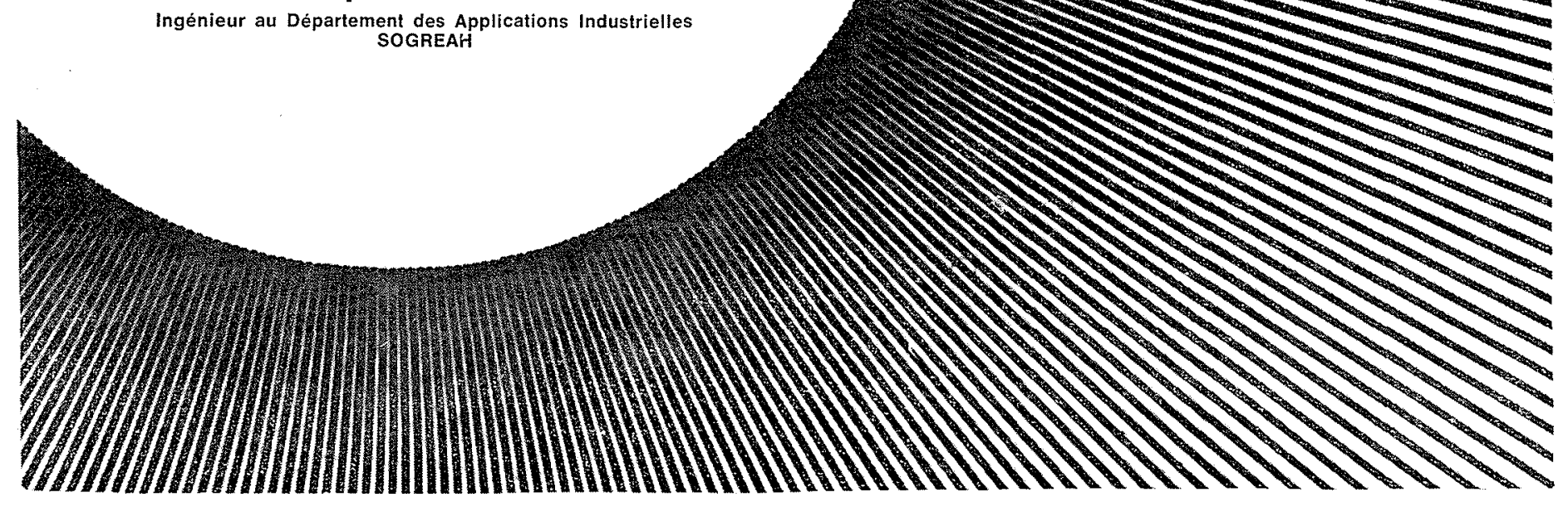

Introduction

Les circuits industriels de gaz comprimés sont parfois équipés de véritables générateurs de pulsations de pression que sont les compresseurs à pistons. Ces circuits de gaz sont eux-mêmes une suite de résonateurs acoustiques où se propagent et peuvent être amplifiées les ondes de pression émises.

Il est donc important d'étudier des remèdes à ces pulsations de pression susceptibles de provoquer des incidents graves, tels des ruptures mécaniques par fatigue, des bris de clapets ou encore une altération du rendement de l'installation.

Propagation des ondes acoustiques dans une conduite Fréquences propres acoustiques

Le problème de la propagation des ondes acoustiques dans un gaz contenu dans une conduite est suffisamment connu pour que nous nous contentions de rappeler brièvement les principes die base.

Une fluctuation de pression $p$ à l'entrée d'une conduite se propage dans le gaz avec la vitesse $c$ :

$$
c=\sqrt{\gamma \frac{\mathrm{RT}}{\mathrm{M}}}
$$

$\mathrm{R}$ étant la constante des gaz parfaits;
M la masse moléculaire du gaz;

$\mathrm{T}$ sa température absolue.

Cette propagation est régie par l'équation suivante:

$$
\frac{\partial^{2} p}{\partial t^{2}}=c^{2} \frac{\partial^{2} p}{\partial x^{2}}
$$

$x$ étant l'abscisse suivant l'axe de la conduite.

Quand la fluctuation de pression atteint l'autre extrémité de la conduite, elle se réfléchit avec ou sans changement de signe, pour se propager ensuite vers l'entrée de la conduite où elle a été engendrée. Lorsque l'onde de pression est sinusoïdale, l'onde réfléchie interfère en tout point de la conduite avec l'onde incidente émise à l'entrée. A certaines fréquences il se forme des ondes stationnaires avec des ventres et des nouds de pulsations. Ces fréquences sont les fréquences propres acoustiques définies par les équations :

$$
f=\frac{\mathrm{K} c}{2 \mathrm{~L}} \quad \text { ou } \quad f=\frac{(2 \mathrm{~K}-1) c}{4 \mathrm{~L}}
$$

suivant que les deux extrémités de la conduite sont ouvertes, ou que l'une des extrémités est fermée.

Simulation analogique

La connaissance de ces fréquences propres acoustiques est à la base de toute étude de pulsations. Leur calcul ne présente aucune difficulté tant que l'on reste dans le cas simple d'une conduite unique (diamètre constant, absence 


\section{P. LEON}

de singularités); il devient rapidement complexe dans les installations réelles où l'on trouve des conduites de diamètres différents, des singularités, des branchements en dérivation, etc. Le recours à une méthode de calcul plus élaborée s'impose, et dans cette optique la simulation analogique est vite apparue intéressante et a fait l'objet d'un certain nombre de réalisations. En effet, il suffit de se rappeler l'équation des télégraphistes régissant la tension électrique $\mathrm{E}$ le long d'une ligne non résistante et sans défaut d'isolement :

$$
\frac{\partial^{2} \mathrm{E}}{\partial t^{2}}=\frac{1}{\mathrm{LC}} \frac{\partial^{2} \mathrm{E}}{\partial x^{2}}
$$

équation semblable à celle de la propagation des ondes acoustiques.

On peut également comparer un circuit électrique série comprenant une self $L$, une résistance $R$ et une capacité $c$ à un résonateur de Helmholtz constitué par une conduite de longureur $l$ et de section $s$ et une cavité de volume V. Le courant $i$ dans le circuit électrique soumis à une tension sinusoïdale a pour valeur:

$$
i=\frac{\mathrm{E}}{\sqrt{\mathrm{R}^{2}+[\mathrm{L} \omega-(1 / \mathrm{C} \omega)]^{i}}} \sin (\omega t-\varphi)
$$

Le débit de gaz $\mathrm{Q}$ dans le résonateur soumis à une pression acoustique sinusoïdale $p$ a pour valeur :

$$
\mathrm{Q}=\frac{p}{\sqrt{\frac{\rho \omega \mathrm{K}}{2 \pi}+\left(\frac{\rho c^{2}}{\mathrm{~V} \omega}-\frac{\rho l}{s} \omega\right)^{2}}} \sin (\omega t-\psi)
$$

Il est, dès lors, très tentant de construire un circuit électrique « analogue » au circuit acoustique et de le soumettre aux excitations électriques correspondantes pour en déduire les caractéristiques du circuit acoustique et sa réponse aux excitations réelles.

\section{Calcul sur ordinateur. Programme. Limitations}

Le calcul digital permet également d'obtenir ces résultats, tout en présentant l'avantage de s'adapter plus facilement à des installations plus complexes. Mais il peut offrir en outre un certain nombre d'avantages sur le simulateur ana. logique en ce qui concerne, par exemple, les non-linéarités. l'influence de la vitesse moyenne de l'écoulement, celle de la température. C'est pourquoi nous avons mis au point deux programmes de calcul sur ordinateur des pulsations de pression dans les circuits de gaz dont voici l'essentiel :

\section{Premier programme.}

Ce programme est une transposition sur ordinateur du simulateur analogique. Il comprend deux parties qui peuvent être dissociées.

- La première partie permet essentiellement de calculer l'amplitude des pulsations de pression et du débit pulsé provoqués par une fluctuation sinusoïdale du débit à l'entrée du circuit. La fréquence excitatrice varie suivant une progression arithmétique, et le régime pulsatoire est calculé pour chacune des fréquences.

Cette première partic du calcul permet donc de connấtre la réponse en fréquence du circuit et, par conséquent, toutes ses fréquences propres acoustiques.

- La deuxième partie est conçue pour les circuits équipés de compresseurs à pistons alternatifs. Le débit du compresseur dans un étage donné est assimilé à une portion de sinusoïde pour chaque période ou demi-période du compresseur (*). Cette portion du sinusoïde, qui est donc périodique est décomposée en série de Fourier.

L'excitation réelle, liée au mouvement du piston, peut dès lors être remplacée par une somme d'excitations sinusoïdales dont les fréquences et les amplitudes ont été calculées par la décomposition en série de Fourier. Le programme utilise ensuite les résultats de la première partie pour calculer le régime pulsatoire dû à chacune de ces composantes sinusoïdales de l'excitation réelle. Il fait enfin la somme, à chaque instant, de ces différents régimes pulsatoires pour obtenir en chaque point l'évolution de la pression et du débit en fonction du temps.

Ce programme est applicable à des circuits ramifiés ou maillés. Les temps de calcul sont très petits, puisque le temps complet de calcul sur ordinateur IBM 360-65 est de l'ordre de deux minutes.

La première partie du programme présente des avantages particuliers. En effet, cette partie peut être dissociée et constituer un programme indépendant. Ceci permet une étude directe de l'influence sur les fréquences propres acoustiques de paramètres tels que la longueur d'une conduite ou le volume d'un ballon.

On peut utiliser également cette partie de programme pour calculer les fréquences acoustiques de n'importe quel circuit, quelle que soit la source d'excitation. Dans le cadre de l'étude d'un circuit équipé de compresseurs dont les fréquences d'excitation sont connues, cette première partie permet de mieux estimer un risque réel de pulsations lorsqu'une fréquence d'excitation est voisine d'une fréquence propre. En effet, dans ce cas, le calcul de la deuxième partie peut ne pas faire apparaître de pulsations dangereuses mais il suffirait d'une petite erreur, toujours possible, sur la vitesse de propagation pour que ces deux fréquences se confondent, ce qui augmenterait très rapidement le niveau des pulsations.

Mais ce programme présente aussi certaines limitations. Tout d'abord, il est limité au domaine linéaire. En effet, il suppose que la vitesse de propagation des ondes est constante en un point donné, quelle que soit l'amplitude des ondes de pression et, par conséquent, l'échauffement qui en résulte localement. Il suppose aussi que les pertes de charge sont linéaires, c'est-à-dire proportionnelles au débit. D'autre part, ce programme ne prend pas en compte l'influence de la vitesse moyenne de l'écoulement sur la vitesse de propagation des ondes par rapport à la conduite; mais dans la plupart des cas, cette vitesse moyenne est faible vis-à-vis de la vitesse de propagation.

\section{Deuxième programme}

Ce deuxième programme est basé sur la méthode des caractéristiques utilisée pour l'étude des coups de bélier dans les conduites hydrauliques. L'équation différentielle de la propagation des ondes de pression admet la solution générale:

$$
p=f\left(t+\frac{x}{c}\right)+g\left(t-\frac{x}{c}\right)
$$

Cette solution permet de déterminer, à partir d'une situation initiale connue, l'évolution des pressions dans le temps jusqu'à l'obtention d'un régime périodique qui caractérise le fonctionnement de l'installation. On obtient ainsi

(*) Nota. - Le calcul doit être fait séparément pour chaque étage du compresseur: le programme ne prend pas en compte une interaction éventuelle entre les pulsations de deux étages voisins. 
les courbes de variation de pression (et de débit) en tous les points désirés du circuit. On obtient également, dans le cas de circuits excités par des compresseurs à piston, le diagramme PV permettant de calculer le rendement du compresseur. Enfin, une analyse des fluctuations de pression en chaque point fournit les amplitudes et les phases du fondamental et des harmoniques. Cette méthode est plus précise que la précédente, car elle n'est pas astreinte à toutes les conditions de linéarité. On peut ainsi exprimer les pertes de charge proportionnellement au carré des vitesses. Cependant, la propagation des ondes est toujours soumise à une loi linéaire.

Ce deuxième programme permet également de représenter n'importe quelle loi de fonctionnement des singularités. Cet avantage est particulièrement intéressant pour étudier l'influence du mouvement des clapets d'aspiration et de refoulement des compresseurs à piston ou celle de tout autre organe de réglage.

Ce deuxième programme conduit à des temps de calcul sur ordinateur IBM $360-65$ plus longs que le précédent et qui peuvent varier de 10 à 20 minutes suivant que le calcul converge plus ou moins vite vers une solution périodique reproductible avec une certaine approximation.

En fait, ces deux programmes sont complémentaires, le premier permettant une étude paramétrique conduisant à la meilleure solution, le deuxième permettant un calcul plus précis des fluctuations ainsi que l'étude de l'influence des singularités.

\section{Circuits industriels. Conséquences des pulsations}

Les compresseurs à piston ayant pour but de comprimer du gaz dans des circuits industriels sont de véritables générateurs de pulsations de pression, étant donné qu'ils injectent (ou prélèvent) périodiquement dans le circuit pendant un temps très court un certain débit de gaz. Les circuits eux-mêmes, composés de tuyauteries à branchements multiples (parfois en dérivation), de ballons ayant des fonctions diverses (stockage, séparation), d'échangeurs de chaleur, de singularités (vannes de réglage, diaphragmes de mesure, coudes) constituent un ensemble de résonateurs acoustiques et ont, par conséquent, un grand nombre de fréquences propres acoustiques. Les effets de cette association « excitateur-résonateur » sont évidents et du même genre que ceux que l'on obtient dans tout système mécanique soumis à une excitation périodique ou aléatoire: ce sont les amplifications des pulsations émises lorsque les conditions de la résonance sont remplies ou même approchées. I.es conséquences de ces pulsations et de leur amplification éventuelle sur les circuits industriels de gaz comprimés sont les suivantes:

- mise en vibration des tuyauteries dont l'une des fréquences propres mécaniques peut correspondre à la fréquence des pulsations, et rupture par fatigue des parties les plus sollicitées;

- altération du rendement du compresseur, du fait de l'influence des ondes de pression sur le fonctionnement des clapets d'aspiration ou de refoulement;

- rupture des clapets eux-mêmes, sollicités anormalement par les fluctuations de pression;

- enfin, conséquences diverses sur des circuits particuliers, par exemple les circuits appelés réacteurs dans la pétrochimie, telles que l'incidence sur la fabrication d'un produit, sur la régulation d'un débit, etc.
Etude des remèdes

L'étude des remèdes doit être envisagée en fonction des caractéristiques de l'excitation d'une part (fréquences et amplitudes des ondes de pression émises), et celles des circuits récepteurs d'autre part (fréquences propres acoustiques et mécaniques).

La première partie du premier programme de calcul permet de déterminer les fréquences propres acoustiques du circuit. Quant aux fréquences propres mécaniques, leur calcul ne présente pas de difficulté majeure si l'on dispose d'un ordinateur.

L'excitation des compresseurs à piston peut être assimilée à une impulsion périodique de débit. $\mathrm{La}$ décomposition en série de Fourier de cette impulsion périodique assimilable à une portion de sinusö̈de fait apparaître une fréquence fondamentale, égale à la fréquence du compresseur multipliée par le nombre d'effets (deux en général), et un certain nombre d'harmoniques dont les impairs sont les plus importants.

La deuxième partie du premier programme de calcul permet de calculer les amplitudes et les phases de ces composantes.

Compte tenu de ces différentes données, deux remèdes principaux peuvent être utilisés, soit séparément, soit simultanément suivant les cas particuliers qui peuvent se présenter :

- utilisation de ballons antipulsatoires;

- déplacement d'une ou de plusieurs fréquences propres acoustiques.

\section{Utilisation de ballons antipulsatoires.}

Ces ballons sont conçus suivant le principe des filtres acoustiques ayant pour propriété d'atténurer fortement les ondes de pression dans certaines bandes de fréquences. Ils sont habituellement constitués de deux ballons d'expansion réunis par un tube de faible section. L'atténuation $\mathrm{A}$ est définie par:

$$
\mathrm{A}=20 \log \frac{\mathrm{V}_{i}}{\mathrm{~V}_{i r}}
$$

$\mathrm{V}_{i}$ étant l'onde incidente;

$\mathrm{V}_{t}$. l'onde transmise.

L'atténuation, en fonction de la fréquence, revêt l'allure générale donnée par les courbes de la figure 1. On remarque sur ces courbes une première zone de fréquence de très faible atténuation suivie de plusieurs larges zones de forte atténuation. La fréquence séparant la zone de faible atténuation de la première zone de forte atténuation s'appelle la fréquence de coupure.

Le premier remède consiste donc à équiper les compresseurs de ballons antipulsatoires à l'aspiration et au refoulement. Ces ballons sont dimensionnés en fonction des fréquences excitatrices, et du niveau de pulsations acceptable en aval des ballons. Ce niveau est fixé en général à $\pm 1 \%$ de la pression moyenne.

Ballons antipuisatoires
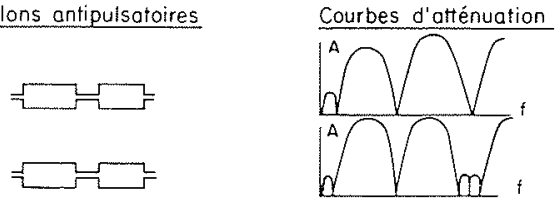

$1 /$ 


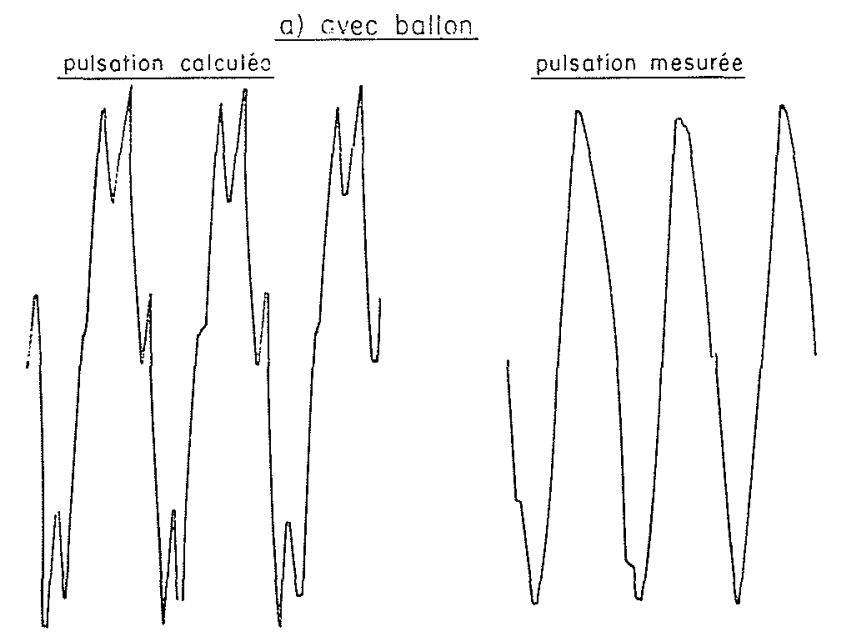

b) sans ballon
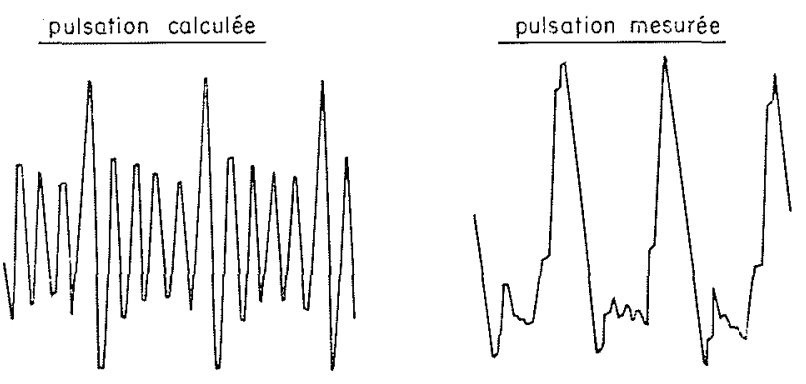

2/ Pulsations de pression.
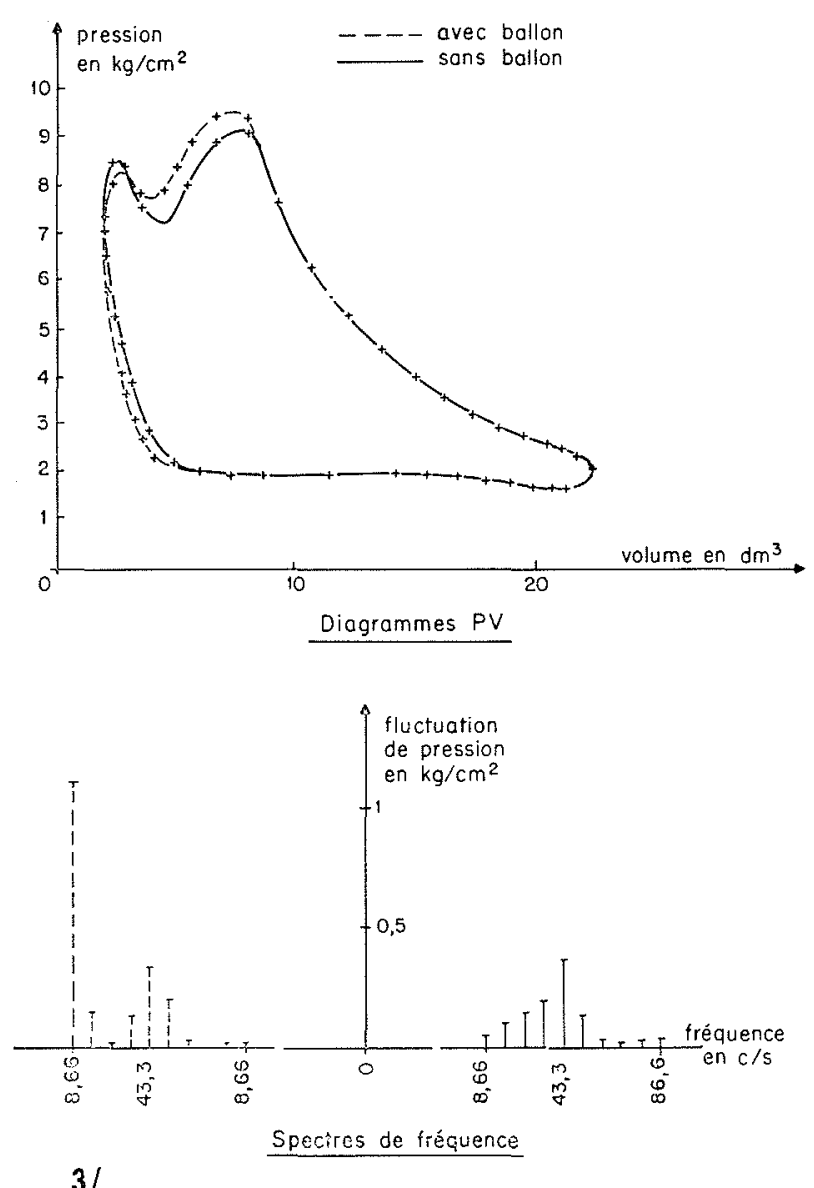

31
Les caractéristiques $\mathrm{d} u$ ballon et son efficacité peuvent facilement être obtenues, et éventuellement modifiées, au moyen du premier programme de calcul.

\section{Déplacement des fréquences propres acoustiques.}

Le deuxième remède consiste à modifier une ou plusieurs fréquences propres acoustiques du circuit lorsque ces fréquences sont très proches des fréquences excitatrices.

Les fréquences propres acoustiques dépendant des dimensions géométriques des organes auxquelles elles se rapportent, il suffit de changer ces dimensions pour modifier la fréquence. Dans le cas d'une conduite, par exemple, on peut, soit augmenter sa longueur, soit intercaler une capacité à mi-longueur de manière à la remplacer par deux conduites de longueur moitié. En fait, il s'agit là d'un travail de tâtonnement dont le résultat doit être vérifié par un calcul.

\section{Remarques.}

Dans la plupart des cas, seul le premier remède, c'est-àdire l'équipement du compresseur en ballons antipulsatoires, est appliqué. Cette solution est, en général, satisfaisante, mais elle peut comporter un risque lorsque l'une des fréquences propres acoustiques du circuit correspond à l'une des fréquences d'excitation. En effet, bien qu'atténuée par le ballon, cette excitation peut subsister à la sortie du ballon avec une amplitude égale à $\pm 1 \%$ de la pression moyenne. En cas de résonance bien marquée, l'amplification dynamique peut être suffisante pour que la pulsation devienne préjudiciable au fonctionnement de l'installation.

On ne peut que déplorer l'habitude prise trop souvent d'étudier les ballons antipulsatoires en fonction des compresseurs seuls et en ignorant complètement le circuit. On demande, en effet, aux constructeurs de compresseurs d'équiper ceux-ci de ballons et de garantir un niveau maximum de pulsations de $\pm 1 \%$ de la pression moyenne. Or une étude globale prenant en compte le compresseur, les ballons et les circuits montrerait sans doute que ce niveau maximum de $\pm 1 \%$ pourrait être souvent augmenté sans préjudice pour l'installation, ce qui conduirait à une réduction des dimensions des ballons. Cette étude globale mettrait également en évidence les risques de résonance dont nous avons parlé plus haut.

\section{Exemples}

Un premier exemple est relatif à une installation de compression d'acide chlorhydrique sur laquelle un ballon était monté en dérivation à la sortie du compresseur. Des mesures de pulsations de pression ont mis en évidence l'influence néfaste de ce ballon. Un calcul des pulsations venait confirmer ce phénomène. La figure 2 représente les pulsations enregistrées et calculées, avec et sans ballon. La figure 3 représente les diagrammes PV ainsi que les spectres de fréquence des fluctuations obtenues au moyen du deuxième programme de calcul. On retrouve la fréquence fondamentale du compresseur égale à $8,66 \mathrm{~Hz}$, ainsi que le cinquième harmonique égale à $43,3 \mathrm{~Hz}$.

Un deuxième exemple est relatif à un incident qui s'est produit sur une tuyauteria de refoulement d'un hypercompresseur d'éthylène. Le niveau élevé de la pression de refoulement, soit 2500 bars, ne permettant pas l'installation d'un ballon antipulsatoire, une tuyauterie de faible section était montée directement au refoulement du compresseur. Cette 
tuyauterie, soumise à d'importantes vibrations, se fissurait assez régulièrement après un temps de fonctionnement variant de quelques heures à quelques jours.

Une campagne de mesures de pulsations de pression et de vibrations a montré que l'une des fréquences propres acoustiques du circuit de refoulement était en accord avec la fréquence propre mécanique du premier mode de flexion de la tuyauterie de refoulement. Les impératifs de la production exigeaient un dépannage rapide. Nous avons donc cherché à amortir les vibrations mécaniques de la tuyauterie sans agir sur l'excitation. Un amortisseur dynamique, fixé sur la tuyauterie elle-même, de fréquence propre égale à celle de la tuyauterie, a permis de réduire de plus de $60 \%$ l'amplitude des vibrations. Cette réduction a été suffisante pour éviter les ruptures par fatigue.

Une deuxième solution, du type acoustique, a consisté à diminuer la fréquence propre acoustique en augmentant la longueur de la tuyauterie de refoulement. La fréquence propre mécanique étant fonction du carré de la longueur et la fréquence acoustique étant fonction de la longueur on évitait ainsi facilement le risque de résonance. Cette solution a été appliquée avec succès sur une autre installation identique qui était alors en projet.

\section{Conclusion}

Les pulsations de pression engendrées par des organes tels que les compresseurs à piston, dans les circuits industriels de gaz comprimés, peuvent avoir des conséquences graves comme la rupture des tuyauteries ou celle des clapets du compresseur. Elles peuvent aussi avoir des conséquences, peut-être moins spectaculaires, mais également importantes, sur le plan économique ou fonctionnel, par exemple la diminution du rendement d'un compresseur.

La protection des circuits par l'installation de ballons antipulsatoires, à l'aspiration et au refoulement des compresseurs est en général satisfaisante. Toutefois, l'étude de ces ballons ne devrait se concevoir qu'en fonction des caractéristiques acoustiques de toute l'installation, et non seulement en fonction de celles du compresseur. Une étude globale peut montrer tout l'intérêt, et parfois la nécessité, de modifier l'une des fréquences propres acoustiques du circuit, ce qui constitue un second remède aux conséquences des pulsations de pression.

Ces études peuvent être faites en utilisant des programmes de calcul sur ordinateur conçus plus spécialement pour les circuits comprenant des compresseurs à piston, mais que l'on peut également utiliser pour d'autres circuits.

Discussion

Président: M. HanNothiaux

M. le Président remercie M. LÉon qui, par une approche mathématique, éclaire le mécanisme de phénomènes encore mal expliqués. Sur une question de $M$. le Président, M. LÉon précise que le premier programme de calcul digital étant lié au calcul analogique, présente, comme ce dernier, l'inconvénient de linéariser les phénomènes. Le deuxième permet de prendre en compte la non-linéarité des pertes de charge (proportionnelles au carré de la vitesse) et d'in troduire l'influence du clapet de refoulement et d'autres clapets sur les pulsations dans le circuit.

Personne ne demandant à intervenir, M. le Président donne la parole à $M$. Daubert pour l'exposé de sa communication.

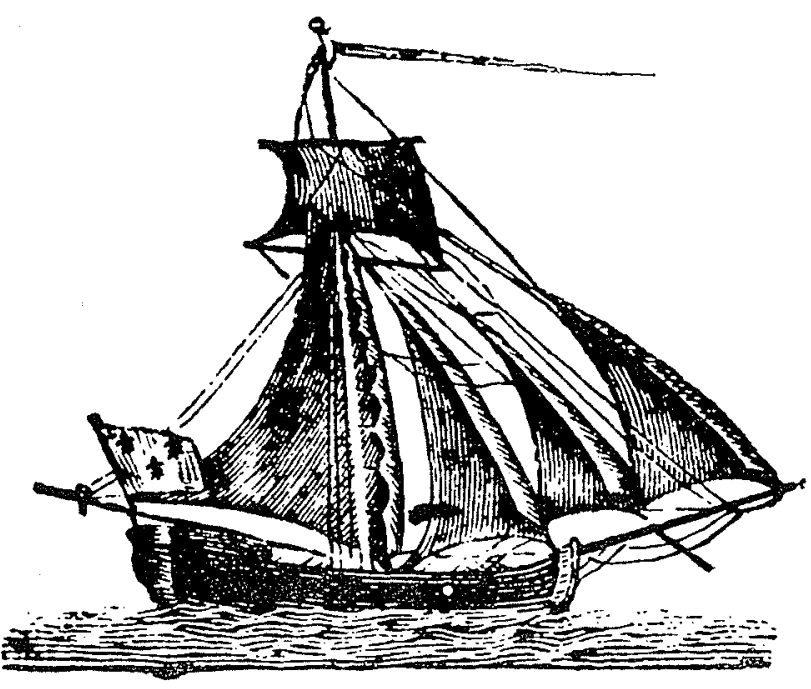




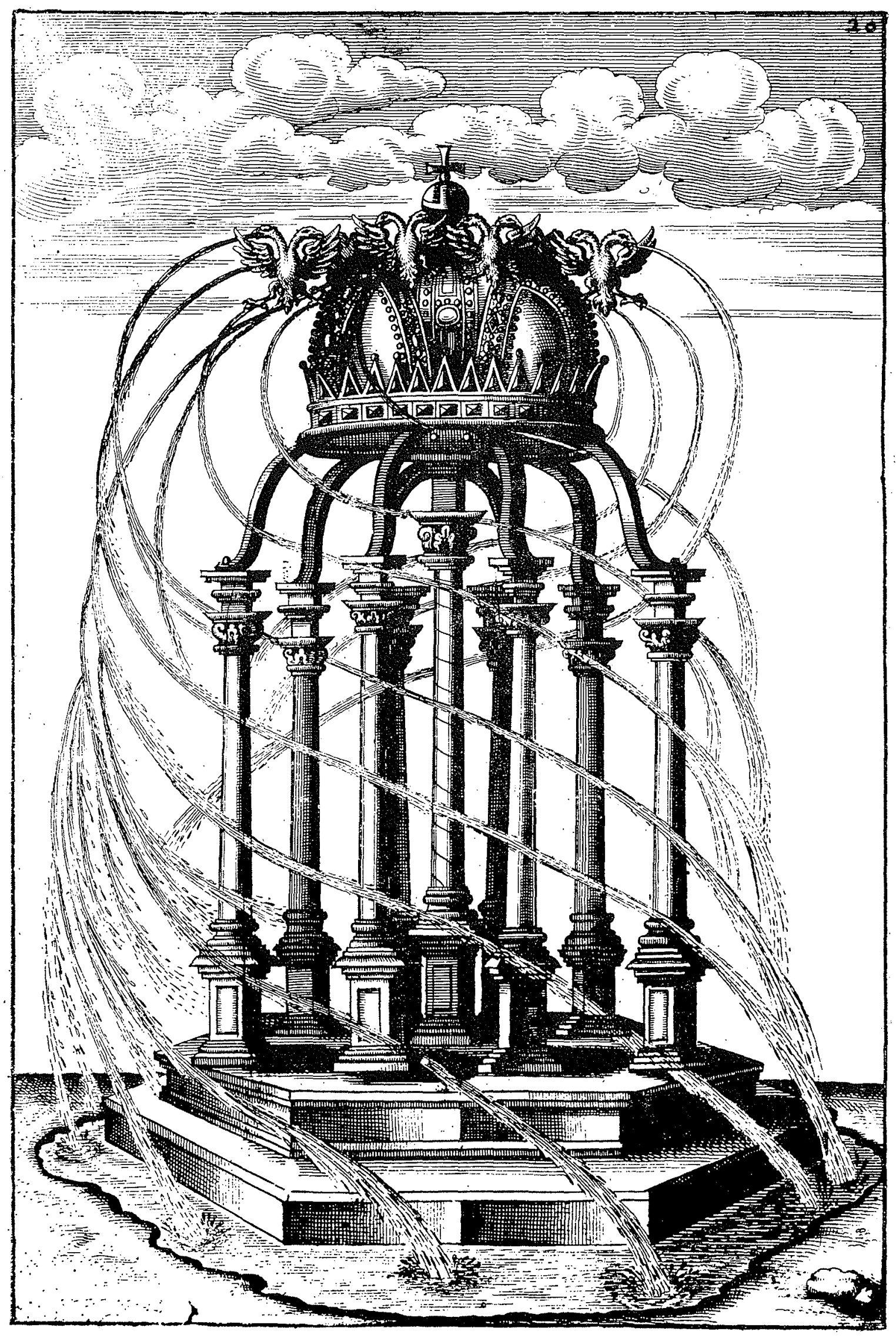

